\title{
Article \\ Decreased Physical Activity during Pregnancy Is Associated with Excessive Gestational Weight Gain
}

\author{
Jia-Jing Sun ${ }^{1,2}$ and Li-Yin Chien ${ }^{3, *}$ (1) \\ 1 School of Nursing, College of Medicine, National Taiwan University, Taipei 112304, Taiwan; \\ c22881@gmail.com \\ 2 Department of Nursing, Heping Fuyou Branch of Taipei City Hospital, Taipei 112304, Taiwan \\ 3 Institute of Community Health Care, College of Nursing, National Yang Ming Chiao Tung University, \\ Yang-Ming Campus, Taipei 112304, Taiwan \\ * Correspondence: lychien@nycu.edu.tw; Tel.: +886-2-28267142; Fax: +886-2-28238614
}

Citation: Sun, J.-J.; Chien, L.-Y Decreased Physical Activity during Pregnancy Is Associated with Excessive Gestational Weight Gain. Int. J. Environ. Res. Public Health 2021, 18, 12597. https://doi.org/10.3390/ ijerph182312597

Academic Editors: Joachim G. Voss and Sandul Yasobant

Received: 4 November 2021

Accepted: 27 November 2021

Published: 29 November 2021

Publisher's Note: MDPI stays neutral with regard to jurisdictional claims in published maps and institutional affiliations.

Copyright: (c) 2021 by the authors. Licensee MDPI, Basel, Switzerland. This article is an open access article distributed under the terms and conditions of the Creative Commons Attribution (CC BY) license (https:// creativecommons.org/licenses/by/ $4.0 /)$.
Abstract: The majority of pregnant women in Taiwan are not considered physically active. During pregnancy, many women decrease their physical activity levels when compared to pre-pregnancy. The purpose of this study was to examine the association between decreased physical activity from pre-pregnancy to pregnancy and excessive gestational weight gain (GWG). This study applied a prospective panel design. Recruitment was conducted at six medical facilities in Taiwan and lasted from August 2016 to April 2017. Physical activity levels were determined both before and during pregnancy using the International Physical Activity Questionnaire-Short Form, with data subsequently being transformed into METs-min/week. Excessive GWG was determined based on the body mass index (BMI) specific GWG range. We recruited 747 pregnant women in their second trimester and followed them through to one-month postpartum. About $40 \%$ of participants (41.2\%) exhibited excessive GWG. Physical activity decreased from an average of $2261(\mathrm{SD}=3999)$ to 1252 $(\mathrm{SD}=2258)$ METs-min/week from pre-pregnancy to pregnancy $(p<0.0001)$. Controlling for age and pre-pregnancy BMI, a logistic regression model revealed that a decline in physical activity of $>4000$ METs-min/week from pre-pregnancy to pregnancy was associated with an increased risk for excessive GWG $(\mathrm{OR}=2.83,95 \% \mathrm{CI}$ : 1.27-4.43). A substantial decrease in physical activity from pre-pregnancy to pregnancy was a risk factor for excessive GWG. Although most women decreased their physical activity during pregnancy, only those pregnant women who were physically active prepregnancy could show the kind of large decrease that resulted in excessive GWG. Health professionals should continue to develop strategies for counteracting the problematic trend of decreasing PA during pregnancy among low-risk pregnant women.

Keywords: pregnancy; physical activity; gestational weight gain; obesity; maternal

\section{Introduction}

Estimates have shown that there were approximately 38.9 million overweight pregnant women throughout the world in 2014, with 14.6 million falling into the category of obesity [1]. Although there is a lack of recent data on the global burden of overweightness and obesity among pregnant women, estimates suggest that $20 \%$ of women will be obese by 2025 [2]. In this context, maternal obesity has become an increasing area of concern [2]. Looking specifically at Taiwan, a national breastfeeding survey (2012-2016) showed that the proportions of women with overweightness and obesity pre-pregnancy were $10.6 \%$ and $5.9 \%$, respectively [3]. This is a notable issue, as women who are overweight or obese at conception are known to have increased risk of excessive gestational weight gain (GWG) and poor pregnancy outcomes [4].

Evidence also suggests there is an association between excessive GWG and future overweightness/obesity in the offspring [5,6]. Further, mothers with excessive GWG are at higher risk for preterm birth, cesarean delivery [7], and postpartum weight retention [8]. In 
response to the obesity epidemic in the Unites States, the American Institute of Medicine (IOM) defined optimal ranges of total GWG for each category of the pre-pregnancy bodymass index (BMI), as follows: $12.5-18 \mathrm{~kg}$ for underweight, 11.5-16 kg for normal weight, $7-11.5 \mathrm{~kg}$ for overweight, and 5-9 kg for obese women [9]. Weight gains below and beyond the recommended amounts are termed inadequate and excessive GWG, respectively. About $20 \%$ and $48 \%$ of women in the United States experience inadequate and excessive GWG, respectively [10]. The IOM criteria for GWG based on local cut-off for BMI $(18.5,24$, and $27 \mathrm{~kg} / \mathrm{m}^{2}$ for underweight, normal, overweight, and obese, respectively) are adopted by the Taiwan Health Promotion Administration [11]. A national survey of postpartum women in Taiwan reported that the proportion of women with inadequate and excessive GWG was $41.5 \%$ and $22.4 \%$, respectively [12]. Most women actually gain amounts of weight that fall outside the recommendations.

A growing number of studies have examined the efficacy of physical activity (PA) interventions during pregnancy. In this regard, a Cochrane review found no evidence to recommend or discourage exercise during pregnancy in view of its effect on maternal and infant health outcomes [13]. Nonetheless, exercise is often advocated during pregnancy due to its overall health benefits, particularly in preventing chronic diseases and unhealthy weight gain. Furthermore, women with low-risk pregnancies should be able to engage in high-intensity exercise programs that include jogging and aerobic activities, which also help with weight management [14-17]. In short, research has demonstrated that exercise during pregnancy does not harm the fetus, but can provide benefits for both the mother and baby $[14,16]$. On the other hand, pregnant women with low PA may be two to three times more likely to experience excessive GWG [7].

The guidelines suggesting that healthy women who are not already highly active or engaging in vigorous activity should spend at least $150 \mathrm{~min}$ per week walking or participating in moderate-intensity aerobic activities, while women who already engage in vigorous aerobics or large amounts of activity can continue doing so during pregnancy [14-16]. On the other hand, certain aspects of traditional Chinese culture suggest that PA can "disturb the fetus" meaning it should be avoided to prevent miscarriage-like symptoms such as abdominal pain and/or vaginal bleeding [18]. One study found that the median active PA time decreased from $80 \mathrm{~min} /$ week (IQR $=0-240 \mathrm{~min} /$ week) during pre-pregnancy to $0 \mathrm{~min} /$ week (IQR $=0-60 \mathrm{~min} /$ week) during both mid- and late pregnancy, with results also showing low overall active PA levels during pregnancy and significant drops in active PA times between pre- and during pregnancy [19].

Previous studies have suggested that regular exercise during pregnancy can reduce GWG [20-22], while meta-analyses also concluded that engagement in structured moderate physical exercise programs during pregnancy decreases the risk of gestational diabetes mellitus and diminishes maternal weight gain [21,23]. Nonetheless, a few studies have found that PA (or exercise) was not significantly associated with weight gain during pregnancy [24-26]. There was generally a lack of consideration of PA level before pregnancy and the drop in PA level during pregnancy. The literature thus offers no clear or consistent associations between PA and excessive GWG during pregnancy, especially in observational studies. While this may partially be due to the generally high prevalence of low PA during pregnancy, we are therefore not aware of any studies showing that reduced PA during pregnancy is directly associated with GWG. Based on the above and beginning with the hypothesis that excessive GWG is related to decreased PA during pregnancy, this study examined the crude and adjusted associations between decreased PA from pre-pregnancy to pregnancy and excessive GWG.

\section{Methods}

2.1. Design

This study applied a prospective panel design in which women were recruited during their second trimester of pregnancy (gestational weeks 14-27). All participants were 
evaluated during their second and third trimesters and at one-month postpartum using structured questionnaires.

\subsection{Participants and Setting}

Participants were recruited from four hospitals and two clinics in Taipei, Taiwan, and the study lasted from August 2016 to April 2017. Ethical approval for this study was obtained from 4 institutional review boards (Far Eastern Memorial Hospital: 105073-F; Taiwan Adventist Hospital: 105-E-03; Taipei City Hospital: TEHIRB-10505103; Buddhist Tzu Chi General Hospital-Taipei Branch: 05-M01-005). This included adult pregnant women who were receiving prenatal care at their respective locations of recruitment. None had pre-existing conditions or experienced complications during early pregnancy (e.g., habitual abortion, diabetes, high blood pressure, and hyperthyroidism). All pregnancies were considered stable. Participants were required to communicate in Mandarin Chinese. After receiving explanations of the study's purpose, those who agreed to participate signed consent forms and provided research personnel with their contact details, including email addresses, phone numbers, and postal addresses. A total of 985 women met the inclusion criteria, with 800 of those agreeing to participate in this study. Finally, the analysis included 747 women with complete records (successfully followed throughout 1 month postpartum), including information on PA levels and weight. While some were excluded due to missing information $(n=53)$, they did not significantly differ from those with complete information $(n=747)$ in regard to age, education level, work status, and number of pregnancies.

\subsection{Sample Size Considerations}

In the logistic regression model, when the event probability was set at 0.35 , the odds ratio was 1.85 , and the two-tailed alpha was 0.05 , the required sample size to yield a power of 0.80 was 528, based on the sample size calculation using G-Power [27]. Consequently, we considered that the sample size of the study was adequate.

\subsection{Measures}

Study variables included sociodemographic factors (age, work status, education level, and marital status), PA levels, pre-pregnancy BMI, GWG, and parity. Sociodemographic factors, parity, BMI, and pre-pregnancy PA were collected during the second trimester of pregnancy, while PA levels during pregnancy were collected during both the second and third trimesters of pregnancy.

Physical activity levels were determined using the Taiwanese version of the International Physical Activity Questionnaire-Short Form (IPAQ-SF) [28]. This instrument was used to collect self-reported data on the frequency and duration of walking, moderateintensity, and vigorous-intensity PA over the preceding seven-day period. A previous study reported that the total PA recorded by IPAQ-SF was acceptably reliable across three times (on days 1, 8, and 11) with an intra-class correlation coefficient (ICC) of 0.79 among Chinese adults in Hong Kong [29]. There were no significant differences in average PA between IPAQ-SF and PA-log, demonstrating acceptable concurrent validity. IPAQ-SF had good test-retest reliability in measuring time spent in moderate PA, vigorous PA, and moderate and vigorous PA (ICC ranged from 0.81-0.84) among pregnant women. However, concurrent validity between IPAQ-SF and an objective PA measure, SenseWear Armband, in measuring time spent in moderate PA, vigorous PA, and moderate and vigorous PA was low to fair (correlation coefficient 0.08-0.39). The low to fair concurrent validity between IPAQ-SF and objective measures were in line with other self-reported PA questionnaires [30].

We followed IPAQ guidelines stating that only 10 or more min of activity should be counted [31]. We then multiplied the metabolic equivalent values for the specific types of PA (e.g., walking $=3.3$, moderate activity $=4$, vigorous activity $=8$ ) based on the number of min of the activity completed over one week to yield the METs-min/week 
measurements [32]. A previous study showed that moderate to vigorous PA did not differ between participants in their second and third trimesters of pregnancy [19]. Following this, we used the average METs-min during the second and third trimesters of pregnancy to represent PA levels during pregnancy. The Spearman correlation coefficient of total PA between the second and third trimester was $0.28(p<0.001)$ in this study. Pre-pregnancy PA was enquired in the second trimester and the participants were asked to answer their ordinary PA in a typical week before conception based on their recall.

Maternal weight was obtained from each participant's Mother's Handbook or hospital records, while pre-pregnancy BMI was calculated in $\mathrm{kg} / \mathrm{m}^{2}$ based on maternal prepregnancy weight and height in the medical records. We categorized pre-pregnancy BMI using the Taiwanese cutoffs of $18.5,24$, and $27 \mathrm{~kg} / \mathrm{m}^{2}$ for underweight, overweight, and obesity, respectively [11]. Total GWG was calculated by subtracting the pre-pregnancy weight from the weight at delivery, then categorized as excessive or non-excessive if above or below/within the BMI-specific weight gain ranges established by the Institute of Medicine, respectively [33].

\subsection{Data Analysis}

Statistical analyses were conducted using SPSS for Windows Version 24.0 (IBM Corp, Armonk, NY, USA). Participant characteristics were presented using frequencies and percentages for categorical variables, while means and standard deviations (SDs) were used for continuous variables. The crude associations between excessive GWG and PA or other characteristics were examined using the t-test or chi-squared test. The IPAQ group defined 601-4000 METs-min/week as moderate PA and >4000 METs-min/week as high PA [34]. Given those, PA and decline in PA was divided into $<600,600-4000$, and $>4000$ METs-min/week in the analysis. The logistic regression model was used to examine the net association between PA and excessive GWG. We first fit the logistic regression model by entering all variables, then dropped the least significant variable from the model one at a time. This was repeated until all variables in the model were statistically significant, which was determined based on 2-sided $p$ values $<0.05$. Adjusted odds ratios (aOR) and $95 \%$ confidence intervals ( $95 \%$ CIs) were computed for the logistic model results.

\section{Results}

\subsection{Characteristics of the Participants}

Table 1 shows the characteristics of the 747 analyzed study participants. The mean age was $33.1 \pm 4.4$ years (ranging from 20 to 44 ). As shown, more than $70 \%(73.2 \%)$ had university-level educations or higher, and the majority were married (97.2\%). More than half were primiparous (55\%). More than one-fifth were overweight or obese before pregnancy (12.6\% overweight and $8.4 \%$ obese).

Table 1. Participant Characteristics by Excessive Gestational Weight Gain $(n=747)$.

\begin{tabular}{|c|c|c|c|c|}
\hline \multirow{4}{*}{ Sample Characteristics } & \multicolumn{4}{|c|}{ Excessive Gestational Weight Gain } \\
\hline & \multirow{3}{*}{$\begin{array}{c}\text { Total } \\
n(\%) \\
N=747\end{array}$} & No & Yes & \multirow{3}{*}{$p$-Value } \\
\hline & & $n(\%)$ & $n(\%)$ & \\
\hline & & $n=439$ & $n=308$ & \\
\hline \multirow{5}{*}{$\begin{array}{c}20-29 \\
30-39 \\
>40\end{array}$} & & & & \multirow[t]{4}{*}{0.255} \\
\hline & $146(19.5)$ & $80(18.2)$ & $66(21.4)$ & \\
\hline & $562(75.2)$ & $332(75.6)$ & $230(74.7)$ & \\
\hline & $39(5.2)$ & $27(6.2)$ & $12(3.9)$ & \\
\hline & & & & \multirow[t]{5}{*}{0.672} \\
\hline \multirow{4}{*}{ Education } & $126(16.9)$ & $71(16.2)$ & $55(17.9)$ & \\
\hline & $74(9.9)$ & $45(10.3)$ & $29(9.4)$ & \\
\hline & $435(58.2)$ & $252(57.4)$ & $183(59.4)$ & \\
\hline & $112(15.0)$ & $71(16.2)$ & $41(13.3)$ & \\
\hline
\end{tabular}


Table 1. Cont.

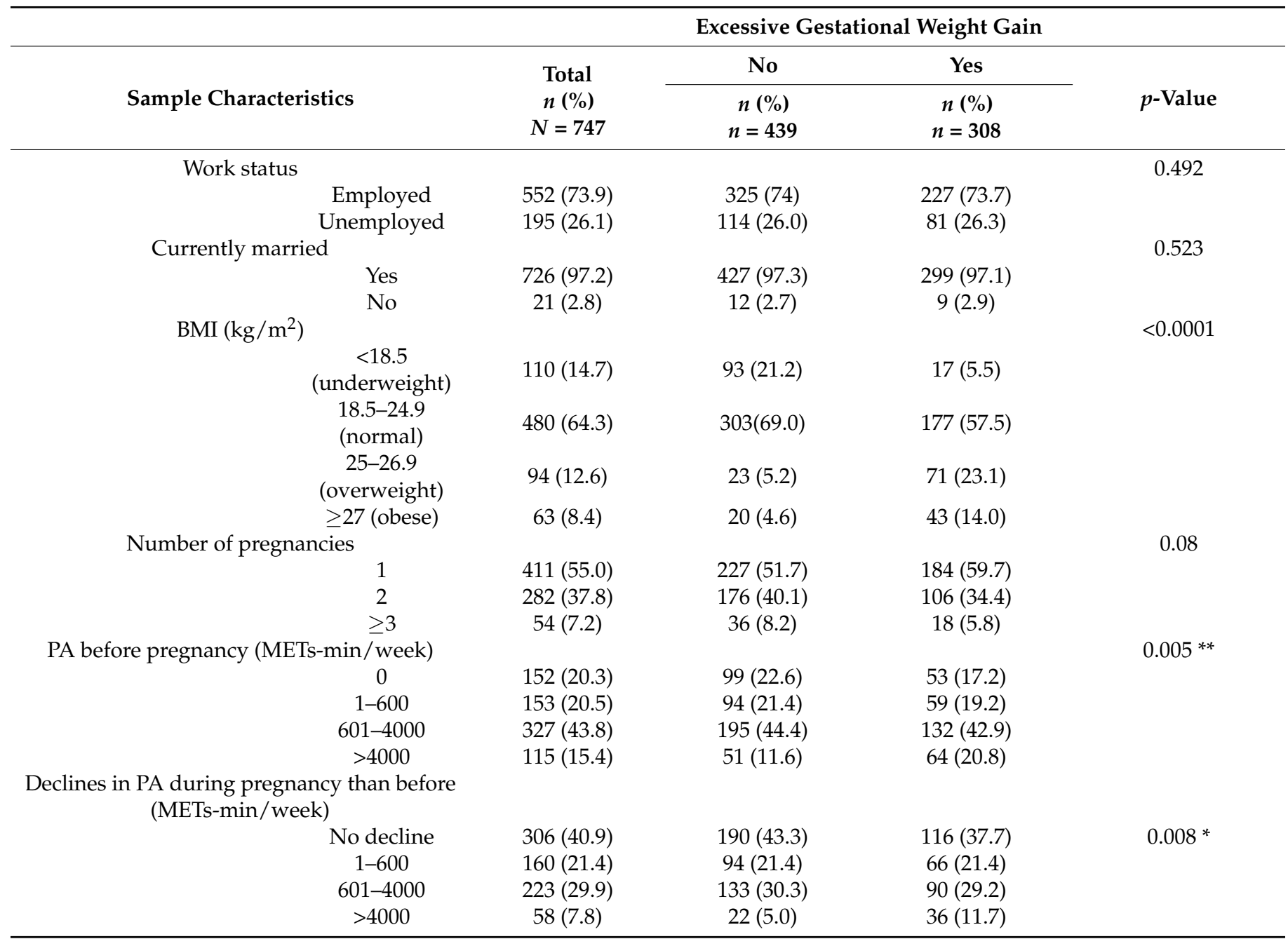

Abbreviations: $\mathrm{BMI}=$ body mass index; $\mathrm{SD}$ = standard deviation; $\mathrm{PA}=$ physical activity. $p$-values were calculated using the chi-squared test or $t$-test ${ }^{*} p<0.05,{ }^{* *} p<0.01$.

\subsection{Physical Activity Levels before and during Pregnancy}

Before pregnancy, $43.8 \%$ and $15.4 \%$ of participants had PA levels of $601-4000$ and $>4000$ METs-min, respectively. About $60 \%$ revealed declines in PA levels during pregnancy when compared to pre-pregnancy levels; declines in PA (METs) were measured at $21.4 \%$, $29.9 \%$, and $7.8 \%$ for decreases of $1-600,601-4000$, and $>4000$ METs-min, respectively (Table 1).

Table 2 shows the proportions of participants who met the common recommended PA guidelines before and during pregnancy. Mean PA levels decreased from $2261 \pm 3999$ before pregnancy to $1252 \pm 2258$ METs-min/week during pregnancy $(p<0.001)$. The proportion of women who reported $\geq 600$ METs-min/week PA decreased from $59.3 \%$ before pregnancy to $44.8 \%$ during pregnancy $(p<0.001)$. In terms of the type of PA, the decline among the proportion meeting vigorous PA $\geq 75 \mathrm{~min} /$ week was more than that of those meeting moderate PA $\geq 150 \mathrm{~min} /$ week ( $21.6 \%$ to $4.6 \%$ for vigorous; $20.9 \%$ to $10.8 \%$ for moderate; both $p<0.001$ ). Walking is the most common type of PA among pregnant women in Taiwan, and is also the type recommended by the Mother's Handbook; the decline in walking $\geq 150 \mathrm{~min} /$ week appeared to be less pronounced ( $44.0 \%$ to $40.2 \%$, $p<0.001$ ). 
Table 2. Physical Activity Before and During Pregnancy $(N=747)$.

\begin{tabular}{|c|c|c|c|c|}
\hline \multirow[b]{2}{*}{ Physical Activity } & \multirow{2}{*}{$\begin{array}{c}\text { Pre-Pregnancy } \\
n(\%)\end{array}$} & \multicolumn{2}{|c|}{ During Pregnancy } & \multirow{2}{*}{$\begin{array}{l}\text { Mean MET } \\
\text { (SD) }\end{array}$} \\
\hline & & & Ts $n(\%)$ & \\
\hline Total physical activity & & $2261(3999)$ & & $1252(2258)$ \\
\hline \multicolumn{5}{|l|}{ Vigorous ( $\geq 75 \mathrm{~min} / \mathrm{wk}$ ) } \\
\hline No & $586(78.4)$ & $1618(2985)$ & $713(95.4)$ & 1150 (2071) \\
\hline Yes & $161(21.6)$ & $4603(5913)$ & $34(4.6)$ & $1625(2815)$ \\
\hline \multicolumn{5}{|l|}{ Moderate $(\geq 150 \mathrm{~min} / \mathrm{wk})$} \\
\hline No & $591(79.1)$ & $1448(2448)$ & $666(89.2)$ & 947 (1702) \\
\hline Yes & $156(20.9)$ & $5340(6488)$ & $81(10.8)$ & $2408(3438)$ \\
\hline \multicolumn{5}{|l|}{ Walking ( $\geq 150 \mathrm{~min} / \mathrm{wk}$ ) } \\
\hline No & $418(56.0)$ & 857 (2535) & $447(59.8)$ & $690(1504)$ \\
\hline Yes & $329(44.0)$ & 4045 (4743) & $300(40.2)$ & 1967 (2794) \\
\hline \multicolumn{5}{|l|}{$\geq 600$ METs-min/wk } \\
\hline No & $304(40.7)$ & $170(201)$ & $412(55.2)$ & $438(679)$ \\
\hline Yes & $443(59.3)$ & 3696 (4679) & $335(44.8)$ & $1811(2743)$ \\
\hline
\end{tabular}

The comparisons of PA level at pre-pregnancy to pregnancy were performed using the chi-squared test or Student's $t$-test. All $p$ were $<0.001$ except for the mean METs between pre- and pregnancy at "Walking ( $\geq 150 \mathrm{~min} / \mathrm{wk})$ No," where $p=0.03$. Abbreviations: METs $=$ METs-min $/$ week; $\min =\min ; \mathrm{wk}=$ week.

The mean PA level was compared before and during pregnancy stratified by whether PA guidelines were met (Table 2). The mean METs-min/week appeared to be higher prepregnancy compared to during pregnancy (all $p<0.001$ ) except for one. For those whose PA was <600 METs-min/week, their mean METs increased from 170 (before pregnancy) to 438 METs-min/week (during pregnancy; $p=0.03$ ).

Declines in PA levels during pregnancy differed significantly by pre-pregnancy PA level (Table 3). Participants with higher pre-pregnancy PA levels showed greater declines during pregnancy than those with lower PA levels. For each pre-pregnancy PA level, about half of participants experienced substantial decreases during pregnancy when compared to their pre-pregnancy levels $(50.4 \%, 54.4 \%$, and $47.7 \%$ of those with $>4000,601-4000$, and 1-600 METs-min before pregnancy had decreases of $>4000,601-4000$, and 1-600 METs-min during pregnancy, respectively). For participants who were totally inactive before pregnancy $(\mathrm{METs}=0)$, PA levels actually increased during pregnancy, although this was $<600$ METs-min/week.

Table 3. Declines in Physical Activity from Pre-pregnancy to Pregnancy Based on Pre-Pregnancy Levels $(n=747)$.

\begin{tabular}{|c|c|c|c|c|c|}
\hline \multirow[b]{2}{*}{ Decline in Physical Activity during Pregnancy } & \multicolumn{5}{|c|}{ Physical Activity Before Pregnancy ${ }^{a}$} \\
\hline & $\begin{array}{c}0 \\
n(\%)\end{array}$ & $\begin{array}{l}1-600 \\
n(\%)\end{array}$ & $\begin{array}{c}601-4000 \\
n(\%)\end{array}$ & $\begin{array}{l}\geq 4000 \\
n(\%)\end{array}$ & Total \\
\hline No decline & $152(100)$ & $80(52.3)$ & $61(19.6)$ & $10(8.7)$ & $306(41)$ \\
\hline $1-600$ & 0 & $73(47.7)$ & $85(26)$ & $2(1.7)$ & $160(21.4)$ \\
\hline $601-4000$ & 0 & 0 & $178(54.4)$ & $45(39.1)$ & $223(29.9)$ \\
\hline$>4000$ & 0 & 0 & 0 & $58(50.4)$ & $58(7.8)$ \\
\hline Total & $152(20.3)$ & $153(20.5)$ & $327(43.8)$ & $115(15.4)$ & 747 \\
\hline
\end{tabular}

$p$-values obtained via Fisher's exact test for frequency comparisons. $p<0.0001$. ${ }^{a}$ METs-min/week.

\subsection{Crude Analysis of Factors Associated with Excessive GWG}

In this study, $41.2 \%(n=308)$ of participants experienced excessive GWG. Table 1 shows the crude associations between participant characteristics and excessive GWG. Of the study variables, pre-pregnancy BMI, pre-pregnancy PA levels, and declined PA during pregnancy were significantly related to excessive GWG. Participants with excessive GWG were significantly more likely to be overweight $(23.1 \%$ versus $5.2 \%)$ and obese (14\% versus $4.6 \%$ ) before pregnancy. Those with excessive GWG showed both a higher mean pre-pregnancy PA level (2881 \pm 4868 versus $1826 \pm 3188$ METs-min, $p=0.001)$ and greater mean decline in PA during pregnancy $(1326 \pm 3142$ versus $785 \pm 2657, p=0.014)$. Using categorized PA levels, $20.8 \%$ of participants with excessive GWG were found to have pre-pregnancy PA levels $>4000$ METS-min, compared to $11.6 \%$ of those without excessive 
GWG. About $11.7 \%$ of participants with excessive GWG showed declines $>4000$ METs-min during pregnancy, compared to 5\% of those without excessive GWG. Other variables were not significantly related to excessive GWG.

\subsection{Multivariate Logistic Regression Model on Factors Associated with Excessive GWG}

Table 4 shows the multivariate regression results. Since pre-pregnancy PA was highly related to declined PA during pregnancy, we decided to use declined PA during pregnancy in the modeling. Further, older age was associated with a decreased odds for excessive GWG. Compared to participants who were younger than 30 years of age, those aged $\geq 40$ and 30-39 were less likely to have excessive GWG (OR = 0.37, 95\% CI: 0.16-0.86 for $\geq 40$; OR $=0.66,95 \%$ CI: 044-0.99 for 30-39). Pre-pregnancy BMI was positively associated with excessive GWG in that the OR for excessive GWG increased as body size increased from normal to overweight/obese comparing to participants who were underweight before pregnancy (ORs = 3.11, 17.29, and 12.71 for normal weight, overweight, and obesity, respectively). After adjusting for age and pre-pregnancy BMI, participants with PA declines >4000 METs-min during pregnancy were 2.38 (95\% CI: 1.27-4.43) times more likely to have excessive GWG than those whose PA levels did not decline from pre-pregnancy to pregnancy. Other levels of decline (1-600, 601-4000 METS-min) were not significantly associated with excessive GWG (though OR estimates for these categories were 1.18 , and 1.13, respectively).

Table 4. Logistic Regression Model for Factors Associated with Excessive Gestational Weight Gain $(n=747)$.

\begin{tabular}{|c|c|c|c|}
\hline & $\mathrm{OR}^{\mathrm{a}}$ & $95 \% \mathrm{CI}$ & $p$-Value \\
\hline \multicolumn{4}{|l|}{ Age (years) } \\
\hline $20-29$ & 1 & & \\
\hline $30-39$ & 0.66 & $(0.44-0.99)$ & $0.045^{*}$ \\
\hline$\geq 40$ & 0.37 & $(0.16-0.86)$ & $0.021 *$ \\
\hline \multicolumn{4}{|l|}{ Pre-pregnancy BMI } \\
\hline Underweight & 1 & & \\
\hline Normal & 3.11 & $(1.78-5.41)$ & $<0.0001^{* * *}$ \\
\hline Overweight & 17.29 & $(8.51-35.15)$ & $<0.0001^{* * *}$ \\
\hline Obese & 12.71 & $(6.00-26.89)$ & $<0.0001^{* * *}$ \\
\hline \multicolumn{4}{|l|}{ Declines in physical activity during pregnancy (METs) } \\
\hline No decline & 1 & & \\
\hline $1-600$ & 1.18 & $(0.77-1.80)$ & 0.441 \\
\hline $601-4000$ & 1.13 & $(0.77-1.66)$ & 0.519 \\
\hline$>4000$ & 2.38 & $(1.27-4.43)$ & $0.006^{* *}$ \\
\hline
\end{tabular}

\section{Discussion}

This study found that declines in PA levels >4000 METs-min/week from pre-pregnancy to pregnancy were positively associated with excessive GWG. While about $60 \%$ of participants decreased their PA levels during pregnancy, only those with high declines (>4000 METs-min/week) showed significant associations with excessive GWG. This finding may not be surprising, since GWG is influenced by many factors other than PA [35]; thus, only substantial declines in PA could retain statistical significance. Further, individuals with reduced levels of PA usually do not exhibit compensatory reductions in energy intake [36,37]. For that reason, a decrease in energy expenditure due to inactivity leads to a positive energy balance, thereby resulting in weight gain [38]. Contrary to previous observational studies, this study found that substantial declines in PA (rather than PA during pregnancy) was associated with excessive GWG. The lack of a significant association between PA and GWG demonstrated by previous observational studies may be due to the fact that PA levels are generally low among pregnant women [24,39]. The recommendations established by the ACOG, CDC, and ACSM assert that healthy women 
who engage in substantial amounts of PA can continue doing so during pregnancy unless there is a medical reason [16]. Based on the recommendations and our results, we suggest that low-risk pregnant women who are physically active before pregnancy should remain so during pregnancy in order to avoid excessive GWG.

There was a significant decline in the mean PA level from pre-pregnancy to pregnancy (2261 (SD 3999) to 1252 (SD 2258) METs-min/week, respectively). There were significant decreases in several types of PA during pregnancy, including vigorous PA, moderateintensity PA, and even walking; based on proportions, however, declines were highest for vigorous $(-17 \%)$, followed by moderate-intensity $(-10.1 \%)$ and walking $(-3.8 \%)$. These results are consistent with a previous study showing that, compared to pre-pregnancy levels, pregnant women experienced significant decreases in total PA levels during their second and third trimesters, including both vigorous and moderate-intensity activity types as well as walking [40]. Reasons for these declines may include self-identified physical limitations and restrictions, a lack of resources, decreased energy, and less time for exercise [41]. Further study is needed to examine reasons for decline in PA levels among pregnant women and to compare whether the reasons differed by their pre-pregnancy PA level and BMI group. Due to the overall benefits, pregnant women need exercise programs that are specifically designed around those barriers. In this regard, special attention should be given to help sustain pre-pregnancy PA levels, especially among women who were considered physically active prior to pregnancy.

Interestingly, we also found that about $40 \%$ of participants increased their PA levels during pregnancy, with those who were previously inactive being more likely to do so (pre-pregnancy 0 METs: $100 \%$; $\leqq 600$ METs: $52.3 \%$ versus $601-4000$ METs: $19.6 \%$; $>4000$ METs: $8.7 \%$; Table 3). Increased PA was mostly found in walking during the third trimester. A previous study found that participating women believed exercise helped them stay in shape and prepare for labor/delivery [42]. This study's results also suggest that the majority of women who are physically inactive before pregnancy may thus be motivated to increase their PA levels while pregnant. Since walking is widely accepted as a beneficial activity among pregnant women, this should be a good way to increase PA levels among women who were not physically active prior to pregnancy. A meta-analysis showed that exercise frequency of three times per week and exercise duration of 30 to 45 min each time can reduce maternal GWG for pregnant women [23]. However, what type and amount of exercise would most benefit pregnant women, or pregnant women with different BMIs or pre-pregnancy PA, remains to be explored by future studies.

More than $40 \%$ of this study's participants experienced excessive GWG, which supports previous findings [7]. This shows that emphasis should still be placed on appropriate weight gain during pregnancy. Further, more than $20 \%$ of participants were overweight/obese before pregnancy, which was also associated with excessive GWG. This also supports previous findings [4]. Health professionals should thus advise pregnant women on healthy GWG during pregnancy, with special attention given to those who are already overweight/obese.

In this study, a younger maternal age ( $<30$ years) was associated with an increased risk of excessive GWG. The finding may be due to the fact that older women may be more concerned with appropriate GWG and smooth pregnancy experiences; as a result, they place greater emphasis on controlling weight gain during pregnancy. However, there is still controversy about whether this factor is generally associated with an increased or decreased risk for excessive GWG [43,44]. Regardless, the association between maternal age and excessive GWG requires additional research.

\section{Limitations}

The study women were from six clinics in a metropolitan area in northern Taiwan, posing concerns to the generalizability of the results. The study participants appeared to be older and more educated than the national data (mean age 32.12 and $58 \%$ with an educational level of university or higher in 2019 [45]). Although pre-pregnancy weights 
and heights were taken from medical records, pre-pregnancy weights were usually selfreported. As such, under- and over-reporting were possible issues. Physical activity was also self-reported; however, previous studies have supported the validity of the IPAQ-SF, which was used in this study [29]. Specifically, the IPAQ-SF enquires about PA during the preceding seven-day period; we averaged the two measures taken during the second and third trimesters to represent PA during pregnancy, since the two measures appeared to be similar. There was a lack of consideration of PA during the first trimester or of potential weekly differences in PA. Pre-pregnancy PA was self-reported in the second trimester and recall bias as well as the accuracy of the report may be a concern. Future studies should collect PA more often and include PA levels collected before pregnancy and during the first trimester in order to gain a more complete picture. In addition, an objective measure of PA such as an accelerometer could be used to further examine the validity of the IPAQ-SF and capture objective PA levels. A smaller effect may not be detected. We did not collect information on the total dietary calorie intake due to the complexity of the measure. Such unmeasured confounders could have influenced our results and should be considered in future studies.

\section{Conclusions}

A decrease in physical activity $>4000$ METs-min/week from pre-pregnancy to pregnancy was found to be a risk factor for excessive GWG. Women with substantial declines in PA from pre-pregnancy to pregnancy are usually those who were physically active prior to becoming pregnant. Health professionals should continue to develop strategies for counteracting the problematic trend of decreasing PA during pregnancy among low-risk pregnant women.

Author Contributions: L.-Y.C. designed and conceived the study. J.-J.S. and L.-Y.C. were involved in the data collection. J.-J.S. analyzed the data. J.-J.S. and L.-Y.C. interpreted the results and wrote the manuscript. All authors have read and agreed to the published version of the manuscript.

Funding: This work was supported by the Ministry of Science and Technology, Taiwan (MOST1042314-B-010-029-MY3). The publication fee for this article was supported by the Ministry of Science and Technology (MOST108-2314-B-010-059-MY3).

Institutional Review Board Statement: This study was approved by institutional review boards at four hospitals (Far Eastern Memorial Hospital: 105073-F; Taiwan Adventist Hospital: 105-E-03; Taipei City Hospital: TEHIRB-10505103; Buddhist Tzu Chi General Hospital-Taipei Branch: 05-M01-005).

Informed Consent Statement: Informed consent was obtained from all subjects involved in the study.

Data Availability Statement: The datasets generated and/or analyzed during the current study are not publicly available due to confidentially reasons, but are available from the corresponding author on reasonable request.

Conflicts of Interest: The authors declare no conflict of interests.

\section{References}

1. Chen, C.; Xu, X.; Yan, Y. Estimated global overweight and obesity burden in pregnant women based on panel data model. PLoS ONE 2018, 13, e202183. [CrossRef] [PubMed]

2. Poston, L.; Caleyachetty, R.; Cnattingius, S.; Corvalán, C.; Uauy, R.; Herring, S.; Gillman, M.W. Preconceptional and maternal obesity: Epidemiology and health consequences. Lancet Diabetes Endocrinol. 2016, 4, 1025-1036. [CrossRef]

3. Waits, A.; Guo, C.Y.; Chang, Y.S.; Chien, L.Y. Dose-response relationships between breastfeeding and postpartum weight retention differ by pre-pregnancy body-mass index in Taiwanese women. Nutrients 2020, 12, 1065. [CrossRef]

4. Liu, L.; Ma, Y.; Wang, N.; Lin, W.; Liu, Y.; Wen, D. Maternal body mass index and risk of neonatal adverse outcomes in China: A systematic review and meta-analysis. BMC Pregnancy Childbirth 2019, 19, 105. [CrossRef]

5. Lau, E.Y.; Liu, J.; Archer, E.; McDonald, S.M.; Liu, J. Maternal weight gain in pregnancy and risk of obesity among offspring: A systematic review. J. Obes. 2014, 2014, 524939. [CrossRef]

6. Ogden, C.L.; Carroll, M.D.; Flegal, K.M. High body mass index for age among US children and adolescents 2003-2006. JAMA 2008, 299, 2401-2405. [CrossRef] 
7. Yong, H.Y.; Mohd Shariff, Z.; Koo, S.J.; Sa'ari, B.; Syurafak, N. Pre-pregnancy body mass index, height and physical activity are associated with rate of gestational weight gain among Malaysian mothers. J. Obstet. Gynaecol. Res. 2016, 42, 1094-1101. [CrossRef]

8. van der Wijden, C.L.; Steinbach, S.; van der Ploeg, H.P.; van Mechelen, W.; van Poppel, M.N. A longitudinal study on the relationship between eating style and gestational weight gain. Appetite 2014, 83, 304-308. [CrossRef] [PubMed]

9. Rasmussen, K.M.; Catalano, P.M.; Yaktine, A.L. New guidelines for weight gain during pregnancy: What obstetrician/gynecologists should know. Curr. Opin. Obstet. Gynecol. 2009, 21, 521. [CrossRef] [PubMed]

10. Weight Gain During Pregnancy. Atlanta at Centers for Disease Control and Prevention (US). Available online: https://www.cdc. gov/reproductivehealth/maternalinfanthealth/pregnancy-weight-gain.htm (accessed on 2 August 2020).

11. Body Mass Index by the Taiwan Health Promotion Administration. Available online: http://health99.hpa.gov.tw/OnlinkHealth/ Onlink_BMI.aspx (accessed on 11 June 2020).

12. Waits, A.; Guo, C.Y.; Chien, L.Y. Inadequate gestational weight gain contributes to increasing rates of low birth weight in Taiwan: 2011-2016 nationwide surveys. Taiwan J. Obstet. Gynecol. 2021, 60, 857-862. [CrossRef]

13. Brown, J.; Ceysens, G.; Boulvain, M. Exercise for pregnant women with pre-existing diabetes for improving maternal and fetal outcomes. Cochrane Database Syst. Rev. 2017, 12, CD012696.

14. Physical Activity and Exercise During Pregnancy and the Postpartum Period by the American College of Obstetricians and Gynecologists. Available online: https://www.acog.org/clinical/clinical-guidance/committee-opinion/articles/2020/04/ physical-activity-and-exercise-during-pregnancy-and-the-postpartum-period (accessed on 14 May 2021).

15. ACSM Health and Fitness Summit by the American College of Sports Medicine. Available online: http://forms.acsm.org/_frm/ Summit2012/pdfs /72\%20Mudd.pdf (accessed on 30 June 2021).

16. Healthy Pregnant or Postpartum Women by the Centers for Disease Control and Prevention (US). Available online: https://www.cdc.gov/physicalactivity/basics/pregnancy/index.htm (accessed on 17 September 2021).

17. Physical Activity Guidelines for Americans by the Disease Prevention and Health Promotion. Available online: https://health gov/our-work/nutrition-physical-activity/physical-activity-guidelines/previous-guidelines/2008-physical-activity-guidelines (accessed on 5 November 2021).

18. Zhang, J.; Savitz, D.A. Exercise during pregnancy among US women. Ann. Epidemiol. 1996, 6, 53-59. [CrossRef]

19. Weng, S.S.; Lee, Y.H.; Chien, L.Y. Physical activity, sitting time and sleep duration before and during pregnancy and pregnancy outcomes: A prospective panel study. J. Clin. Nurs. 2020, 29, 3494-3505. [CrossRef] [PubMed]

20. Lamina, S.; Agbanusi, E. Effect of aerobic exercise training on maternal weight gain in pregnancy: A meta-analysis of randomized controlled trials. Ethiop. J. Health Sci. 2013, 23, 59-64. [PubMed]

21. Sanabria-Martínez, G.; García-Hermoso, A.; Poyatos-León, R.; Álvarez-Bueno, C.; Sánchez-López, M.; Martínez-Vizcaíno, V. Effectiveness of physical activity interventions on preventing gestational diabetes mellitus and excessive maternal weight gain: A meta-analysis. BJOG 2015, 122, 1167-1174. [CrossRef]

22. Streuling, I.; Beyerlein, A.; Rosenfeld, E.; Hofmann, H.; Schulz, T.; von Kries, R. Physical activity and gestational weight gain: A meta-analysis of intervention trials. BJOG 2011, 118, 278-284. [CrossRef]

23. Wang, J.; Wen, D.; Liu, X.; Liu, Y. Impact of exercise on maternal gestational weight gain: An updated meta-analysis of randomized controlled trials. Medicine 2019, 98, e16199. [CrossRef]

24. Chasan-Taber, L.; Silveira, M.; Lynch, K.E.; Pekow, P.; Solomon, C.G.; Markenson, G. Physical activity and gestational weight gain in Hispanic women. Obesity 2014, 22, 909-918. [CrossRef]

25. Han, S.; Middleton, P.; Crowther, C.A. Exercise for pregnant women for preventing gestational diabetes mellitus. Cochrane Database Syst. Rev. 2012, 7, CD009021. [CrossRef]

26. Kramer, M.S.; McDonald, S.W. Aerobic exercise for women during pregnancy. Cochrane Database Syst. Rev. 2006, 3, CD000180. [CrossRef]

27. Faul, F.; Erdfelder, E.; Lang, A.G.; Buchner, A. G* Power 3: A flexible statistical power analysis program for the social, behavioral, and biomedical sciences. Behav. Res. Methods 2007, 39, 175-191. [CrossRef]

28. International Physical Activity Questionnaire Short Form (IPAQ-SF) Taiwanese Version(in Chinese) by the Taiwan Health Promotion Administration. Available online: https: / /www.hpa.gov.tw / Pages / Detail.aspx?nodeid=876\&pid=4900 (accessed on 1 November 2021).

29. Macfarlane, D.J.; Lee, C.C.; Ho, E.Y.; Chan, K.L.; Chan, D.T. Reliability and validity of the Chinese version of IPAQ. J. Sci. Med. Sports 2007, 10, 45-51. [CrossRef]

30. Sanda, B.; Vistad, I.; Haakstad, L.A.H.; Berntsen, S.; Sagedal, L.R.; Lohne-Seiler, H.; Torstveit, M.K. Reliability and concurrent validity of the International Physical Activity Questionnaire short form among pregnant women. BMC Sports Sci. Med. Rehabil. 2017, 9, 7. [CrossRef] [PubMed]

31. Fan, M.; Lyu, J.; He, P. Chinese guidelines for data processing and analysis concerning the International Physical Activity Questionnaire. Zhonghua Liu Xing Bing Xue Za Zhi 2014, 35, 961-964.

32. Lee, P.H.; Macfarlane, D.J.; Lam, T.H.; Stewart, S.M. Validity of the international physical activity questionnaire short form (IPAQ-SF): A systematic review. Int. J. Behav. Nutr. Phys. Act. 2011, 8, 115. [CrossRef] [PubMed]

33. Weight Gain during Pregnancy: Reexamining the Guidelines by the Institute of Medicine and National Research Council. Available online: https:/ / www.cbsnews.com/htdocs/pdf/052809_pregnancy.pdf (accessed on 1 August 2021). 
34. Guidelines for Data Processing and Analysis of the International Physical Activity Questionnaire (IPAQ) by the International Consensus Group. Available online: https://www.researchgate.net/file.PostFileLoader.html?id=5641f4c36143250eac8b45b7 \&assetKey=AS\%3A294237418606593\%401447163075131 (accessed on 2 August 2021).

35. Muktabhant, B.; Lawrie, T.A.; Lumbiganon, P.; Laopaiboon, M. Diet or exercise, or both, for preventing excessive weight gain in pregnancy. Cochrane Database Syst. Rev. 2015, 15, CD007145. [CrossRef]

36. Epstein, L.H.; Roemmich, J.N.; Paluch, R.A.; Raynor, H.A. Influence of changes in sedentary behavior on energy and macronutrient intake in youth. Am. J. Clin. Nutr. 2005, 81, 361-366. [CrossRef]

37. Stubbs, R.J.; Hughes, D.A.; Johnstone, A.M.; Horgan, G.W.; King, N.; Blundell, J.E. A decrease in physical activity affects appetite, energy, and nutrient balance in lean men feeding ad libitum. Am. J. Clin. Nutr. 2004, 79, 62-69. [CrossRef] [PubMed]

38. Ballor, D.L.; Keesey, R.E. A meta-analysis of the factors affecting exercise-induced changes in body mass, fat mass and fat-free mass in males and females. Int. J. Obes. 1991, 15, 717-726.

39. Kraschnewski, J.L.; Chuang, C.H.; Downs, D.S.; Weisman, C.S.; McCamant, E.L.; Baptiste-Roberts, K.; Zhu, J.; Kjerulff, K.H. Association of prenatal physical activity and gestational weight gain: Results from the first baby study. Women's Health Issues 2013, 23, e233-e238. [CrossRef]

40. Downs, D.S.; Chasan-Taber, L.; Evenson, K.R.; Leiferman, J.; Yeo, S. Physical activity and pregnancy: Past and present evidence and future recommendations. Res. Q. Exerc. Sport 2012, 83, 485-502. [CrossRef] [PubMed]

41. Marquez, D.X.; Bustamante, E.E.; Bock, B.C.; Markenson, G.; Tovar, A.; Chasan-Taber, L. Perspectives of Latina and non-Latina white women on barriers and facilitators to exercise in pregnancy. Women Health 2009, 49, 505-521. [CrossRef]

42. Lee, C.; Chiang, I.; Lin, S.; Lin, H.; Hsu, C. Physical activity pattern and related factors among women during pregnancy. Formosan J. Med. 2012, 16, 103-111.

43. Ebrahimi, F.; Shariff, Z.M.; Tabatabaei, S.Z.; Fathollahi, M.S.; Mun, C.Y.; Nazari, M. Relationship between sociodemographics, dietary intake, and physical activity with gestational weight gain among pregnant women in Rafsanjan City, Iran. J. Health Popul. Nutr. 2015, 33, 168-176. [PubMed]

44. Shulman, R.; Kottke, M. Impact of maternal knowledge of recommended weight gain in pregnancy on gestational weight gain. Am. J. Obstet. Gynecol. 2016, 214, e1-e754. [CrossRef] [PubMed]

45. Ministry of the Interior, Taiwan Statistical Report Year 2020 Week 20. Available online: https:/ /www.moi.gov.tw/cp.aspx?n=1169 8https: / / www.moi.gov.tw / cp.aspx?n=11698 (accessed on 18 November 2021). 